\title{
Evaluación del comportamiento agonista de quirquinchos andinos (Chaetophractus vellerosus) en el Zoológico Municipal Vesty Pakos, La Paz, Bolivia
}

\author{
Paola De la Quintana ${ }^{A}$, Grace Ledezma ${ }^{\text {B, } 1}$ y Luis F. Pacheco ${ }^{C}$ \\ A Carrera de Biología, Universidad Mayor de San Andrés, La Paz-Bolivia. E-mail: dcp1186@hotmail.com \\ B Zoológico Municipal Vesty Pakos, Av. La Florida s/n Mallasa, La Paz, Bolivia. E-mail: amzeled@gmail.com \\ ' Instituto de Ecología, Colección Boliviana de Fauna, Universidad Mayor de San Andrés, Campus Universitario, Calle 27 \\ Cota Cota, La Paz, Bolivia. E-mail: luispacheco11@yahoo.com
}

${ }^{1}$ Autor para correspondencia

\begin{abstract}
Resumen El quirquincho andino (Chaetophractus vellerosus) es una de las especies más afectadas por el uso folklórico tradicional en Bolivia (fabricación de charangos, matracas, amuletos y taxidermias), hasta el punto de llevar a esta especie a la categoría En Peligro de extinción en el país. La falta de conocimiento sobre los aspectos conductuales de $C$. vellerosus provoca que algunas acciones del manejo de la especie en cautiverio no sean las más adecuadas. Este estudio se realizó con el objetivo de mejorar el manejo de 12 quirquinchos andinos (siete machos y cinco hembras) en cuanto a su convivencia en el Zoológico Municipal Vesty Pakos y aportar al conocimiento comportamental de estos animales en cautiverio. Se realizó un proceso inicial y gradual de integración entre machos, tomando en cuenta primero el reconocimiento olfativo por un día y luego colocando a cada par de quirquinchos andinos en contacto casi directo a través de una malla por dos días. Se probaron 21 combinaciones y se determinó el grado de dominancia de cada individuo del grupo. Con los resultados obtenidos se hicieron ocho nuevas combinaciones, de las cuales cuatro resultaron exitosas. Se decidió mantener aislado al más agresivo de manera definitiva y formar tres pares con los individuos restantes.
\end{abstract}

Palabras clave: Chaetophractus nationi, etología, interacción, manejo en cautiverio

Evaluation of agonistic behavior of Andean hairy armadillos (Chaetophractus vellerosus) at Municipal Zoo Vesty Pakos, La Paz, Bolivia

Abstract The Andean hairy armadillo (Chaetophractus vellerosus) is one of the species most affected by traditional folkloric use in Bolivia (through the manufacture of charangos, rattles, amulets, and taxidermy). These activities have led to the categorization of this species as Endangered in Bolivia. The lack of knowledge about the behavioral aspects of $C$. vellerosus result in poor husbandry practices in captivity. This study was carried out with the objective of improving the management of 12 armadillos (seven males and five females) in relation to their coexistence at the Municipal Zoo Vesty Pakos, and to contribute to the behavioral knowledge of these animals in captivity. We performed an initial and gradual integration process between males, taking into account olfactory recognition for one day and then placing each pair of armadillos in near direct contact through a mesh for two days. Twenty-one combinations were performed and the most aggressive and tolerant individuals in the group were determined. This resulted in eight new combinations, four of which were successful. The most aggressive animal was isolated definitively and three pairs were conformed with the remaining individuals.

Keywords: captive management, Chaetophractus nationi, ethology, interaction 


\section{INTRODUCCIÓN}

El quirquincho andino (Chaetophractus vellerosus, antes C. nationi; ver Abba et al., 2015) es una de las especies más afectadas por el uso folklórico tradicional en Bolivia, lo cual es la causa principal de que actualmente se encuentre en peligro de extinción en el país (Pérez-Zubieta et al., 2009). Esta especie es cazada intensamente desde hace décadas para ser comercializada con fines tradicionales y artesanales (fabricación de charangos, matracas, amuletos y taxidermias), a pesar de que se hayan ido implementando algunas acciones de protección, como la regulación del control de su tráfico a través de ordenanzas municipales y un mayor control en entradas folklóricas por parte de la Autoridad Ambiental Competente Nacional, contempladas en un Plan de Acción para la Conservación de Mamíferos Amenazados en Bolivia 2014-2018 (MMAyA, 2014). Las poblaciones de quirquinchos andinos habitan en arenales con suelos suaves, donde pueden construir sus madrigueras (Pérez-Zubieta et al., 2009; Pérez-Zubieta, 2011). Si bien al haber pasado a sinonimia con C. vellerosus (Abba et al., 2015) se ha ampliado el conocimiento sobre la biología y ecología de la especie, la cantidad de estudios sobre las poblaciones altoandinas es mínima y la información general sobre la especie es aún escasa; asimismo, la información disponible corresponde principalmente a las poblaciones que no incluían la región altoandina (Carlini et al., 2016). En estado silvestre es una especie solitaria, omnívora y de hábitos tanto diurnos como nocturnos dependiendo de la época del año y la población estudiada (Abba et al., 2009; Noss et al., 2010; Pérez-Zubieta, 2011; Carlini et al., 2016).

La falta de conocimiento de los aspectos conductuales del quirquincho andino podría haber resultado en que su manejo en cautiverio no sea el adecuado, lo que puede incidir en el bienestar de los individuos. Adicionalmente, los estudios en cautiverio pueden aportar información de gran utilidad para el manejo en estado silvestre. Campagna et al. (1984) indican que el comportamiento agonista en el quirquincho grande (C. villosus) tiende a disminuir en intensidad a medida que los individuos tienen más encuentros entre ellos. Esta situación podría indicar que el comportamiento agresivo refleja algún tipo de acostumbramiento o aprendizaje, según la frecuencia de encuentros y la duración de los mismos para establecer relaciones de dominancia. Las observaciones sobre C. villosus (Campagna et al., 1984) sugieren que el quirquincho grande podría ser una especie que se comporte de forma agresiva con sus conespecíficos, al menos en cautiverio, lo cual se ha observado solamente de forma ocasional en quirquinchos andinos alojados en el Zoológico Municipal Andino de la ciudad de Oruro, Bolivia (A. León, com. pers.), y frecuentemente en $C$. vellerosus en el Zoológico Municipal Vesty Pakos (ZMVP) de la ciudad de La Paz, Bolivia (G. Ledezma, obs. pers.).

El objetivo del presente estudio fue explorar diferentes combinaciones de agrupamientos de quirquinchos andinos (C. vellerosus) para encontrar la mejor manera de optimizar el uso de espacio disponible para la especie en el ZMVP. Con los resultados de este estudio se pretende mejorar la convivencia de quirquinchos andinos en cautiverio, disminuyendo las posibilidades de encuentros agresivos entre los individuos.

\section{MATERIALES y MÉTODOS}

\section{Sitio de estudio}

El presente estudio se llevó a cabo en el ZMVP, legalmente establecido y reconocido como Centro de Custodia de Fauna Silvestre por la Autoridad Ambiental Nacional Competente mediante la Resolución administrativa del Viceministerio de Medio Ambiente, Biodiversidad, Cambios Climáticos y de Gestión y Desarrollo Forestal No 34/15. El ZMVP cuenta con un área de $338,30 \mathrm{~m}^{2}$ destinada para los quirquinchos andinos, denominada quirquinchario. Este último está dividido en dos sectores: un área de exhibición (FIG. 1A), que está al aire libre, y otra exclusiva para el manejo que consta de cuatro madrigueras artificiales, situadas por debajo del área de exhibición y que se utilizan para evaluar el estado general de los individuos. Por otro lado, el hospital veterinario del

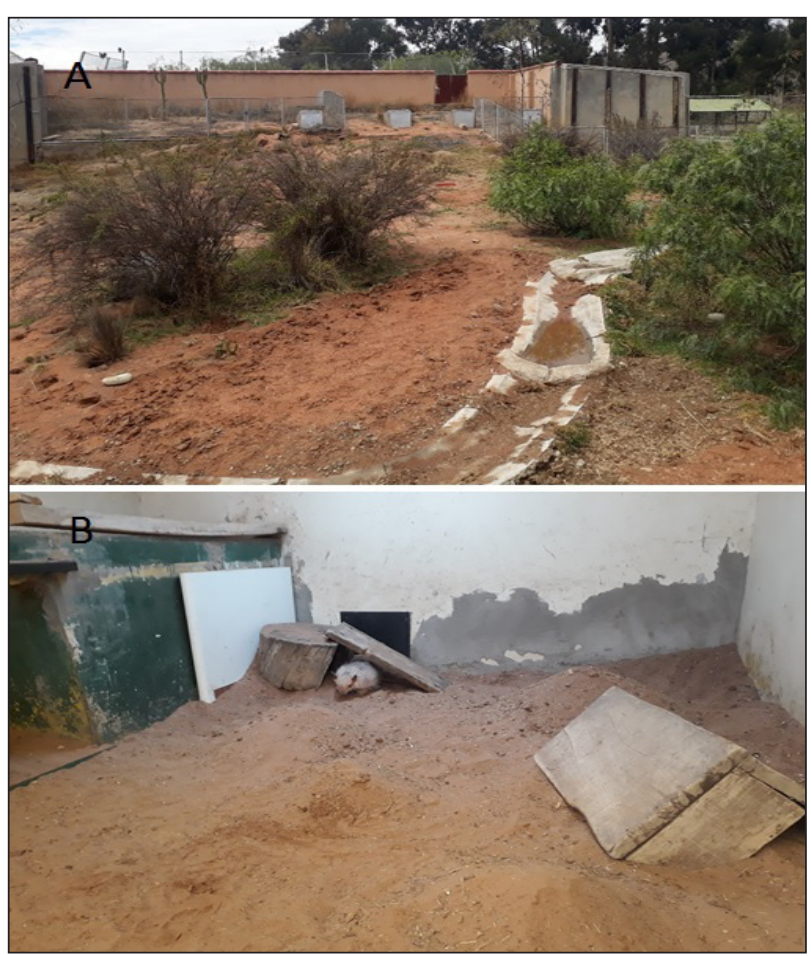

FIGURA 1. A) Ambiente de exhibición de quirquinchos andinos en el Zoológico Municipal Vesty Pakos, La Paz, Bolivia; B) jaula del área de cuarentena. 
TABLA 1. Catálogo general de los comportamientos agonistas de los quirquinchos andinos del Zoológico Municipal Vesty Pakos, La Paz, Bolivia.

\begin{tabular}{|c|c|c|}
\hline Actividad & & Descripción \\
\hline \multirow[t]{3}{*}{ Topetada } & Frontal & $\begin{array}{l}\text { El o los animales utilizan su escudete cefálico para presionarlo en contra del escudete cefálico del } \\
\text { otro individuo. Durante esta topetada se observa una pugna de fuerza entre ambos animales. }\end{array}$ \\
\hline & Lateral & $\begin{array}{l}\text { El quirquincho andino agresivo va contra el flanco del otro individuo empujándolo; el quirquin- } \\
\text { cho andino agredido puede responder con otra topetada o escapar. }\end{array}$ \\
\hline & Caudal & El animal utiliza su escudete cefálico en contra del escudo pélvico del congénere \\
\hline \multirow[t]{2}{*}{ Ataque } & Rasguños & $\begin{array}{l}\text { Empieza con un salto brusco hacia el caparazón o rostro, seguido de movimientos alternantes de } \\
\text { los miembros anteriores y en algunas ocasiones también de los miembros posteriores. }\end{array}$ \\
\hline & Persecución & $\begin{array}{l}\text { Uno de los individuos persigue a otro, no llega a atacarlo con rasguños ya que la malla de divi- } \\
\text { sión los separa. Cuando existe persecución sin malla de división la persecución puede convertirse } \\
\text { en ataque con rasguños, pero no en todos los casos. Se ha podido observar que el individuo } \\
\text { perseguido arroja tierra con las patas traseras hacia el individuo perseguidor. }\end{array}$ \\
\hline Advertencia & Gruñido & $\begin{array}{l}\text { Los individuos emiten un sonido parecido a un bufido mientras su cuerpo vibra; la duración de } \\
\text { este sonido es de unos segundos. }\end{array}$ \\
\hline
\end{tabular}

ZMVP tiene un área de cuarentena que cuenta con 12 ambientes de $4 \mathrm{~m}^{2}$ cada uno (FIG. 1B), todos bajo techo.

Al inicio del estudio, el ZMVP mantenía 12 quirquinchos andinos (siete machos, denominados M1 a M7, y cinco hembras, identificadas como $\mathrm{H} 1$ a H5). Dos hembras y dos machos vivían en el área de exhibición del quirquinchario y los otros ocho individuos (cinco machos y tres hembras) en el área de cuarentena. Cada quirquincho andino del área de cuarentena estaba aislado en un cubil diferente, por las peleas continuas entre ellos a lo largo del año que llegaban a producir lesiones cutáneas, algunas leves y otras que presentaban pérdida de piel. Para la parte experimental del trabajo (ver más adelante), los cuatro quirquinchos andinos del quirquinchario fueron trasladados al área de cuarentena.

\section{Etapa de acostumbramiento}

El estudio empezó en septiembre de 2011. Inicialmente se procedió a identificar y marcar a los individuos. Se usaron etiquetas de diferentes colores, colocadas en la frente y en la región caudal del caparazón, para identificar cada individuo. La parte experimental del estudio se realizó en cinco ambientes del área de cuarentena, cada uno de $4 \mathrm{~m}^{2}$, cuyas paredes laterales eran de ladrillo revestido y una pared frontal de malla olímpica metálica en la que se encontraba una puerta del mismo material. Tres de los ambientes fueron divididos por la mitad con malla olímpica metálica, sostenida firmemente a un marco de madera, de manera que cada una de las seis jaulas resultantes tenía $2 \mathrm{~m}^{2}$; dos ambientes quedaron sin modificar. Al piso de cada jaula se le colocó arena roja arcillosa como sustrato para ofrecer un ambiente adecuado a los quirquinchos andinos en el cual podían construir sus madrigueras.

En base a la experiencia reportada en el Zoológico Municipal Andino de Oruro sobre la convivencia pacífica entre hembras de la especie (I. Rodríguez, com. pers.), se juntó a las cinco hembras en una sola jaula, sin divisiones y sin acostumbramiento previo, y se les observó durante dos días con la confianza de que no presentaran comportamientos agonísticos entre ellas. En el caso de los machos el proceso de integración fue gradual. Para ello se tomó en cuenta que el reconocimiento entre los individuos de C. villosus está basado en información química, percibida por el olfato mediante comportamientos de acercamiento hocico-pelviano, hocico-rostral y hocico-genital (Campagna et al., 1984), y se asumió que C. vellerosus se comportaría de manera similar.

En una primera etapa, que fue denominada de integración, se extrajeron heces y parte del sustrato (arena roja), que se supuso tenían el olor de cada uno de los siete quirquinchos andinos machos, reconocible por los otros individuos. Estos materiales fueron colocados dentro de las jaulas de los otros individuos, según el individuo con el cual le tocaría luego interactuar. De esta manera, cada uno podría establecer contacto indirecto con los demás a través del olfato. Se repitió este procedimiento cada tres días durante 27 días. Una segunda etapa (acostumbramiento) se llevó a cabo en las jaulas previamente divididas. En cada lado se colocó a un individuo para permitir el contacto visual a través de la malla de separación por dos días continuos, bajo el supuesto que esto podría reducir la posibilidad de que surgieran comportamientos agonistas al momento de juntarlos. En cada ciclo de interacción el individuo que quedaba sin pareja se quedó solo en otra de las jaulas sin modificar. Este procedimiento se repitió con todos los pares de quirquinchos a lo largo de esos 27 días, de manera que los siete quirquinchos andinos machos tuvieron contacto olfativo y visual a través de la malla de separación. Cabe notar que los tiempos para cada fase 
de trabajo se ajustaron arbitrariamente, de acuerdo a conversaciones con el personal del ZMVP.

\section{Seguimiento conductual durante la etapa de acostumbramiento}

El primer día que se sometió a los individuos a los rastros de olor de los otros quirquinchos andinos (durante la primera etapa de integración) se observó al grupo completo a través de muestreos de barrido (scan sampling) cada 10 minutos (Martin \& Bateson, 1993), repitiendo este protocolo desde las 08:00 hs hasta las 16:00 hs. Con este procedimiento se obtuvo un etograma base en el que se definieron los comportamientos más comunes y aquellos más útiles para esta investigación. El etograma completo será detallado en otra publicación. Durante la etapa de acostumbramiento se dio seguimiento al comportamiento mediante observación directa de los individuos por dos noches continuas (mientras permanecían con sus respectivos pares en las jaulas divididas), por el método de muestreo focal escalonado (Martin \& Bateson, 1993). Se centró la atención sobre un par de individuos a lo largo de la sesión (10 min) y se registraron todas las conductas observadas que denotaban actividad, aunque para fines de este estudio sólo se analizaron las conductas agonistas que se describen en la TABLA 1. Este procedimiento fue realizado cada hora, desde las 14:00 hs hasta las 08:00 hs, y repetido al día siguiente. Posteriormente se realizó un ajuste al horario de los seguimientos, iniciando a las 11:00 hs y concluyendo a las 21:00 hs, debido a que en los primeros dos días de muestreo se verificó que los quirquinchos andinos presentaban poca actividad a partir de las 21:00 hs.

Terminado el proceso de acostumbramiento, se realizó una evaluación sobre el grado de aceptación de unos quirquinchos andinos con los otros, aún separados por las mallas. Para esta evaluación se utilizó como indicador de agresividad el porcentaje de comportamientos agonistas (TABLA 1) a través del cerco, respecto al total de conductas registradas por cada uno de los quirquinchos andinos (las cuales se contabilizaron sólo con fines comparativos). Con estos datos se confeccionó la TABLA 2 para cuantificar el contraste entre la agresión infringida y recibida por cada individuo. Como resumen se realizó una sumatoria de cada uno de los datos para determinar el índice de agresión, distinguiendo la recibida de la infringida por cada individuo.

\section{Conformación de grupos de machos}

En esta tercera etapa se escogieron pares de machos que, durante la etapa de acostumbramiento, no presentaron un índice de agresión alto entre ellos. Dependiendo de la respuesta comportamental de cada par (indicador de agresión) se analizaron las posibilidades de formar pares fijos de machos para optimizar el uso de espacio en el ZMVP. Se consideró que un índice de agresión $\geq 11 \%$ define pares de individuos agresivos. En contraposición, los pares de individuos que tenían $<11 \%$ de índice de agresión se consideraron "tolerantes". No se halló un dato que permitiera establecer un porcentaje fundamentado

TABLA 2. Porcentaje del comportamiento agonista que presentaron los pares de machos de Chaetophractus vellerosus con respecto a otros tipos de comportamiento. * Pares de machos que se sometieron a la tercera etapa.

\begin{tabular}{|c|c|c|c|c|c|c|c|c|}
\hline & M1 & M2 & M3 & M4 & M5 & M6 & M7 & $\begin{array}{l}\text { Promedio de } \\
\text { agresiones infringidas }\end{array}$ \\
\hline \multirow[t]{2}{*}{ M1 } & & $5,1^{*}$ & 13,5 & $8,57^{*}$ & $6^{*}$ & 6,06 & $8,57^{*}$ & 7,97 \\
\hline & & $(\mathrm{n}=273)$ & $(\mathrm{n}=518)$ & $(\mathrm{n}=245)$ & $(\mathrm{n}=350)$ & $(\mathrm{n}=231)$ & $(\mathrm{n}=245)$ & \\
\hline \multirow[t]{2}{*}{ M2 } & $10,9^{*}$ & & 16,3 & 15,8 & 24,7 & 14,5 & $8^{*}$ & 15,04 \\
\hline & $(\mathrm{n}=448)$ & & $(\mathrm{n}=301)$ & $(\mathrm{n}=399)$ & $(\mathrm{n}=539)$ & $(\mathrm{n}=385)$ & $(\mathrm{n}=175)$ & \\
\hline \multirow[t]{2}{*}{ M3 } & 10,8 & 16 & & 15,9 & $4,26^{*}$ & 25 & 11,4 & 13,93 \\
\hline & $(\mathrm{n}=455)$ & $(\mathrm{n}=259)$ & & $(\mathrm{n}=308)$ & $(\mathrm{n}=329)$ & $(\mathrm{n}=280)$ & $(\mathrm{n}=245)$ & \\
\hline \multirow[t]{2}{*}{ M4 } & $7,69^{*}$ & 8,3 & 16,7 & & $7,14^{*}$ & 5,63 & $1,28^{*}$ & 7,79 \\
\hline & $(\mathrm{n}=273)$ & $(\mathrm{n}=252)$ & $(\mathrm{n}=252)$ & & $(\mathrm{n}=294)$ & $(\mathrm{n}=497)$ & $(\mathrm{n}=1092)$ & \\
\hline \multirow[t]{2}{*}{ M5 } & $10,2^{*}$ & 11 & $4,92^{*}$ & $11^{*}$ & & 15,2 & 13,6 & 11,03 \\
\hline & $(\mathrm{n}=413)$ & $(\mathrm{n}=441)$ & $(\mathrm{n}=427)$ & $(\mathrm{n}=315)$ & & $(\mathrm{n}=322)$ & $(\mathrm{n}=308)$ & \\
\hline \multirow[t]{2}{*}{ M6 } & 21,3 & 13 & 16,7 & 16,1 & 26,2 & & 14,3 & 17,98 \\
\hline & $(\mathrm{n}=329)$ & $(\mathrm{n}=420)$ & $(\mathrm{n}=336)$ & $(\mathrm{n}=434)$ & $(\mathrm{n}=294)$ & & $(\mathrm{n}=294)$ & \\
\hline \multirow[t]{2}{*}{ M7 } & $7,5^{*}$ & $3,7^{*}$ & 13,5 & $0,78^{*}$ & 4,26 & 7,5 & & 6,21 \\
\hline & $(\mathrm{n}=280)$ & $(\mathrm{n}=189)$ & $(\mathrm{n}=259)$ & $(\mathrm{n}=903)$ & $(\mathrm{n}=329)$ & $(\mathrm{n}=280)$ & & \\
\hline
\end{tabular}




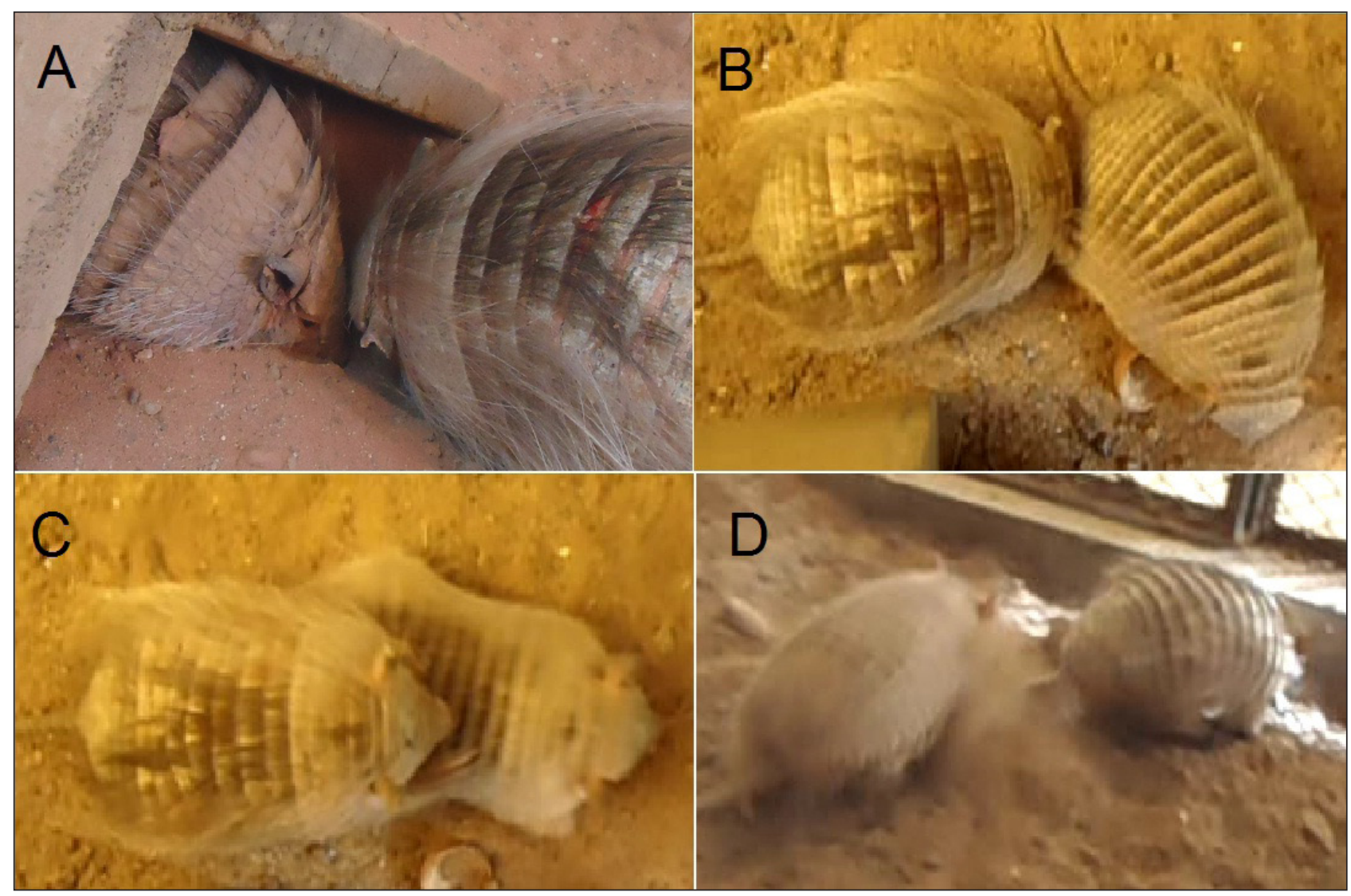

FIGURA 2. Comportamientos agonistas registrados en quirquinchos andinos machos mantenidos en el Zoológico Municipal Vesty Pakos, La Paz, Bolivia. A) Topetada frontal; B) topetada caudal; C) rasguños; D) persecución. Para la descripción de los comportamientos ver TABLA 1.

luego de una búsqueda exhaustiva en la literatura, por lo cual establecimos el criterio de $11 \%$ de forma arbitraria pero siguiendo nuestras percepciones, tomando en cuenta la intensidad y frecuencia de los comportamientos agonistas.

Los individuos identificados como "tolerantes" fueron reunidos por pares en una jaula, sin malla de separación. Las observaciones iniciaron al momento de dejarlos juntos en la jaula, empleándose nuevamente el método de muestreo focal escalonado y manteniendo el horario (de 11:00 a 21:00 hs) por tres días continuos. En esta etapa se mantuvieron a los pares en contacto directo, mientras no presentaran agresiones considerables; es decir, si ocurrían ataques con potencial de ocasionar lesiones serias se reducía el tiempo de interacción o se cancelaba inmediatamente la prueba con ese par de quirquinchos andinos.

\section{RESULTADOS}

\section{Comportamiento de hembras}

Las cinco hembras que se pusieron juntas, sin previo proceso de acostumbramiento, no presentaron agresiones entre ellas durante las primeras horas. Ocasionalmente las hembras $\mathrm{H} 2$ y $\mathrm{H} 5$ se perseguían mutuamente o a otras hembras, aunque sin atacarlas. El segundo día de interacción se las observó durmiendo a todas juntas y no se registraron más comportamientos de persecución.

\section{Machos agresivos y sumisos en la etapa de acostumbramiento}

Los quirquinchos andinos machos tuvieron reacciones distintas y presentaron comportamientos agonistas en diferentes niveles con cada macho con el que interactuaron a través de la malla (TABLA 1, FIG. 2). El quirquincho andino M6 fue identificado como el más agresivo, con porcentajes de agresión entre 13\% y $26,2 \%$ con los distintos machos. El menos agresivo fue M7; solamente se mostró agresivo con M3. Por otro lado, M3 resultó ser el más agredido, ya que algunos de los quirquinchos andinos se mostraron más agresivos con este individuo que con el resto (TABLA 2). Todo esto nos brindó mejores pautas sobre qué individuos podrían ser más tolerantes con otros, y por tanto permitió establecer qué pares de quirquinchos andinos podrían convivir en la misma jaula.

\section{Comportamiento entre pares de machos al compartir jaula}

Las pruebas de interacciones entre quirquinchos andinos machos fueron realizadas con seis de los siete que existían en el ZMVP. De los ocho pares de machos puestos a prueba, cuatro fueron exitosos en términos de baja agresividad: M5-M3, M2-M7, M1-M7 
y M4-M1. De esa manera, se logró la conformación de tres pares de machos que podían convivir sin presentar eventos agonistas de consideración. Si bien M7 podía hacer par tanto con M2 como con M1, se decidió juntarlo con M2 por dos motivos: i) M1 logró habituarse con M4 (el cual era agresivo con el resto de los individuos) y ii) cuando se separó a M7 de M2, ambos se mostraron inquietos, rondando permanentemente la cerca hasta que M7 trepó la cerca para pasar al lado de M2 en tres ocasiones.

\section{Monitoreo de pares y grupos establecidos posterior al traslado}

$\mathrm{Al}$ concluir esta parte del estudio (diciembre de 2011), se decidió mantener a M6 aislado de manera definitiva, colocar a los pares M4-M1 y M3-M5 en dos jaulas en el área de cuarentena y llevar a las cinco hembras junto al par de machos M2-M7 al quirquinchario. Antes de colocar a los individuos en el recinto, se les inocularon microchips subcutáneos (MS Schippers Passion for Farming, modelo MICRO ID/12 ISO Transponder, Schippers Europe BV, London, United Kingdom) para poder identificarlos permanentemente, ya que las marcas puestas al principio del estudio eran temporales.

De enero a agosto del año 2012, las observaciones de los quirquinchos andinos fueron realizadas por los guardafaunas de forma oportunista, puesto que no es fácil observarlos porque suelen pasar buena parte del tiempo escondidos en sus madrigueras. Durante ese periodo, no se detectaron señales de agresión entre los individuos. En septiembre de 2012, por la necesidad de evaluar el estado físico y sanitario, se realizó un seguimiento mediante observaciones continuas durante dos semanas. En este periodo se capturó a los individuos que se divisaban y se los aisló temporalmente en las madrigueras del área de manejo, hasta concluir con la captura y revisión física de todos los individuos. En esta etapa del seguimiento se verificó el buen estado de los dos machos y se hallaron cuatro nuevos individuos juveniles (tres machos y una hembra) de aproximadamente dos meses de edad. A la vez se observó que dos de las hembras adultas ( $\mathrm{H} 2$ y H3) presentaban las glándulas mamarias turgentes y con presencia de leche, lo cual sugiere que estaban en periodo de lactancia. Las otras tres hembras presentaban buen estado general, pero no se observaron indicios de que estuvieran en lactancia.

A partir de este evento de reproducción, se aisló a las hembras de los machos en otro recinto como método de control reproductivo reversible, y se dividió el quirquinchario en tres compartimentos. En cada compartimento se colocó a los pares de machos que estuvieron conviviendo en cuarentena por 10 meses continuos sin agredirse.

El monitoreo de salud de los quirquinchos andinos se realizó desde entonces cada tres meses. Acorde a lo aprendido en este estudio, se fueron conformando nuevos pares e incluso grupos de tres o cuatro machos tolerantes entre sí, según se recibían nuevos quirquinchos andinos en el ZMVP.

\section{DISCUSIÓN}

Según el Reglamento para la Custodia Responsable de Fauna Silvestre (MMAyA, 2017) de Bolivia, se requiere una superficie mínima de $50 \mathrm{~m}^{2}$ para albergar quirquinchos andinos, con una densidad máxima de 1 individuo $/ 25 \mathrm{~m}^{2}$. El quirquinchario del ZMVP cumple con holgura esas especificaciones, pero no así los ambientes del área de cuarentena donde se los mantiene temporalmente por motivos sanitarios y de manejo y en los cuales un periodo prolongado de permanencia podría afectar el bienestar de estos animales. Sin embargo, es notable que los pares de machos que estuvieron conviviendo en una superficie de solamente $2 \mathrm{~m}^{2}$ en el área de cuarentena por un lapso de cinco meses, y las cinco hembras en sólo 4 $\mathrm{m}^{2}$ durante el mismo periodo, no mostraron signos de estrés, se mantuvieron clínicamente sanos y su condición corporal fue buena. De acuerdo a estas observaciones, el área mínima estipulada en el Reglamento posiblemente está sobre-estimada y, al menos temporalmente, es posible albergar quirquinchos andinos en superficies menores, cuidando que no se mantengan juntos, individuos que son agresivos entre ellos. Obviamente, será muy importante que la superficie permita a los quirquinchos andinos moverse con soltura, cavar sus madrigueras en un sustrato apropiado y que cuenten con el recurso alimenticio suficiente para cada uno (Superina et al., 2014).

Por otro lado, Cortés Duarte et al. (2016) indican que la calidad del ambiente es más importante que el tamaño, pues ayuda a que los animales no desarrollen comportamientos anormales y estén más tranquilos. Dentro de los comportamientos normales de los quirquinchos está el cavar túneles, tanto para abrigarse como para esconderse. Si el recinto no brinda esta oportunidad se crea estrés en el individuo, generando problemas de salud, comportamientos agonistas, estereotipados y problemas de reproducción (Cortés Duarte et al., 2016). El área de cuarentena contaba con un colchón de arena que permitía que los quirquinchos andinos cavaran (aunque solo superficialmente), y refugios de madera, lo que posiblemente ayudó a reducir el estrés.

Estudios en Argentina reportan que C. vellerosus hace un uso diferencial del tiempo, teniendo una actividad mayor de día en estación fría y más nocturna durante la estación cálida (Abba et al., 2011). Otro estudio en Bolivia, con quirquinchos andinos en su hábitat natural, no reporta registros de actividad durante el día y determina que la especie es nocturna (Pérez-Zubieta, 2008). En cambio, Zúñiga \& Araya (2014) reportan que la mayor actividad de C. vellerosus en el norte de Chile es en el día, desde las 8:00 hs hasta las 20:00 hs, independientemente 
de la estación. En estudios anteriores en el ZMVP, Molina (2009) reportó que los quirquinchos andinos tenían hábitos principalmente diurnos, mientras que Ledezma (2011) observó que los quirquinchos andinos del ZMVP también tenían actividad nocturna. Estas observaciones previas y las hechas durante este estudio, sugieren que la actividad de los quirquinchos en el ZMVP de La Paz es principalmente diurna, siendo las hembras las que cesan su actividad más tarde por la noche (hasta las 23:00 hs ocasionalmente). Sin embargo, la actividad de los quirquinchos andinos en cautiverio puede estar influenciada por la ausencia de depredadores, como fue observado en osos hormigueros (Myrmecophaga tridactyla; Orlando \& Fernández, 2014), y también por el horario en que se les proporciona alimento. Tomando en cuenta los resultados de este estudio, los reportes de actividad en vida silvestre y las contradicciones que se dan entre distintos estudios, es razonable postular que esta especie adapta sus horarios a las condiciones y retos que su ambiente le pueda proporcionar.

Una de las observaciones más importantes de este estudio fue que las cinco hembras del ZMVP lograron acostumbrarse muy rápidamente a vivir todas juntas. Esto sugiere que las agresiones se dan principalmente entre machos. Bajo esta hipótesis y el hecho que todas las especies de la familia son en general de hábitos solitarios (Noss et al., 2010), es posible predecir que la estructura poblacional en campo podría reflejar similitudes a lo observado en cautiverio, es decir, hembras compartiendo ámbitos de hogar relativamente solapados y machos con ámbitos de hogar más separados y con poco contacto entre ellos. Las observaciones en campo de Pagnutti et al. (2014) apoyarían esta hipótesis, ya que los machos de C. vellerosus tenían un ámbito de hogar grande que abarcaba el de las hembras reproductivas.

Campagna et al. (1984) postulan que los individuos de C. villosus pueden aprender a identificar a su contrincante, y que utilizan la relación de dominancia establecida en encuentros pasados con el mismo animal para modular su comportamiento en futuros encuentros. Nuestros resultados apoyan parcialmente esa hipótesis dado que, por ejemplo, M5 y M3 presentaron comportamiento bastante agresivo al principio del estudio, pero llegaron a tolerarse al pasar el tiempo. Al parecer, este acostumbramiento estuvo mediado por el proceso de interacción a través de la malla de separación. Por otro lado, M4 y M2 tuvieron un nivel de agresión bajo el primer día de interacción directa, pero las agresiones incrementaron a partir del segundo día hasta que esos individuos no se toleraban y tuvieron que ser separados. En ese sentido un par de individuos lograron tolerarse antes de tener encuentros agresivos, mientras que la interacción directa del otro par de individuos no pudo establecer una dominancia que reduzca la agresividad sino que la empeoró. Estas observaciones sugieren que la forma en que los encuentros entre individuos afectan su posterior tolerancia, dependerá de los individuos involucrados y no será posible predecir cómo va a resultar la relación, hasta ponerla a prueba. Esto apunta nuevamente a que no es recomendable albergar quirquinchos andinos juntos, sin antes hacer pruebas bajo supervisión.

Abba et al. (2011) indican que los machos de C. vellerosus son más agresivos durante la época reproductiva. Dado que en este estudio se logró conformar pares de machos que se toleraban todo el año, se puede esperar que estos pares mantengan la convivencia de forma permanente y no se produzcan agresiones ligadas a una época de reproducción, siempre y cuando no se los exponga a la presencia de hembras en época reproductiva y no se los separe. De ser así se debería iniciar con el proceso de acostumbramiento nuevamente.

\section{CONCLUSIONES}

El trabajo de acostumbramiento y posterior puesta a prueba de pares de quirquinchos andinos permitió determinar que el comportamiento agonista de $C$. vellerosus se produce con mayor frecuencia entre algunos individuos, y que algunos pares de individuos pueden convivir sin agredirse. Esto y la confirmación de que las hembras no se agredieron, permitió recomendar una distribución de individuos que hizo más eficiente el uso del espacio en el ZMVP.

Los quirquinchos andinos machos pueden acostumbrarse y tolerar la presencia de otro macho, pero es necesario realizar pruebas de compatibilidad entre individuos para minimizar la probabilidad de agresiones y optimizar el uso del espacio.

\section{AgRADECIMIENTOS}

Al personal de Zoológico Municipal Andino de Oruro y a los guardafaunas del Zoológico Municipal Vesty Pakos por la colaboración durante las observaciones comportamentales de los individuos en horarios poco convenientes. Agradecemos a José Carlos Pérez-Zubieta, Fabián Beltrán, Andrea Morales y especialmente a Erika Cuéllar, Mariella Superina, Agustín Abba y los revisores anónimos por su revisión $\mathrm{y}$ aportes a versiones anteriores de este manuscrito.

\section{REFERENCIAS}

Abba, A. M., S. F. Vizcaíno \& M. Cassini. 2009. Etoecología y conservación de tres especies de armadillos (Dasypus hybridus, Chaetophractus villosus y C.vellerosus) en el noreste dela provincia de Buenos Aires, Argentina. Edentata 8-10: 4147. https: / / doi.org/10.1896/020.010.0101

Abba, A. M., G. Cassini, M. Cassini \& S. F. Vizcaíno. 2011. Historia natural del piche llorón 
Chaetophractus vellerosus (Mammalia: Xenarthra: Dasypodidae). Revista Chilena de Historia Natural 84: 51-64. https://doi.org/10.4067/ S0716-078X2011000100004

Abba, A. M., G. Cassini, G. Valverde, M. Tilak, S. F. Vizcaíno, M. Superina \& F. Delsuc. 2015. Systematics of hairy armadillos and the taxonomic status of the Andean hairy armadillo (Chaetophractus nationi). Journal of Mammalogy 96: 673-689. https://doi.org/10.1093/ jmammal/gyv082

Campagna, C., M. R. Papini \& J. M. Affanni. 1984. El comportamiento agresivo intraespecífico del armadillo Chaetophractus villosus, en condiciones de laboratorio. Revista Latinoamericana de Psicología 16: 443-458.

Carlini, A. A., E. Soibelzon \& D. Glaz. 2016. Chaetophractus vellerosus (Cingulata: Dasypodidae). Mammalian Species 48: 73-82. http: / / dx.doi.org/10.1093/mspecies/ sew008

Cortés Duarte, A., F. Trujillo \& M. Superina. 2016. Behavioral responses of three armadillo species (Mammalia: Xenarthra) to an environmental enrichment program in Villavicencio, Colombia. Zoo Biology 35: 304-312. http: / / dx.doi.org/10.1002/ zoo.21305

Ledezma, G. 2011. Planificación preliminar para el traslado de quirquinchos Chaetophractus nationi a quirquinchario. Zoológico Municipal Vesty Pakos, La Paz, Bolivia. 15 pp.

Martin, P. \& P. Bateson. 1993. Measuring behaviour, an introductory guide. Second Edition. Cambridge University Press, Cambridge, UK. https: / / doi.org/10.1017 / CBO9781139168342

MMAyA - Ministerio de Medio Ambiente y Agua. 2017. Reglamento para la custodia responsable de fauna silvestre. Estado Plurinacional de Bolivia. 33 pp.

Molina, M. 2009. Segunda fase de adaptación de quirquinchos. Informe Cite/GMLP/UMD/ ZOO/BIO/039/2009. Zoológico Municipal Vesty Pakos, La Paz, Bolivia. 5 pp.

Noss, A., E. Cuéllar, H. Gómez, T. Tarifa \& E. AliagaRossel. 2010. Dasypodidae. Pp. 173-212 in:
Distribución, ecología y conservación de los mamíferos medianos y grandes de Bolivia (R. B. Wallace, H. Gómez, Z. R. Porcel \& D. I. Rumiz, eds.). Centro de Ecología Difusión Simón I. Patiño. Santa Cruz de la Sierra, Bolivia.

Orlando, C. \& G. Fernández. 2014. Respuesta antidepredatoria de osos hormigueros (Myrmecophaga tridactyla) mantenidos en cautividad. Edentata 15: 52-57. https://doi. org / 10.5537/020.015.0108

Pagnutti, N., J. Gallo, M. Superina, S. F. Vizcaíno \& A. M. Abba. 2014. Patrones estacionales de distribución espacial y área de acción del piche llorón, Chaetophractus vellerosus (Cingulata: Dasypodidae), en Magdalena, Buenos Aires, Argentina. Mastozoología Neotropical 21:59-65.

Pérez-Zubieta, J. C. 2008. Algunos aspectos de la historia natural del quirquincho andino en hábitats aledaños a tres localidades de la provincia Sur Carangas, Oruro, Bolivia. Tesis de grado, Universidad Mayor de San Simón, Cochabamba. 95 pp.

Pérez-Zubieta, J. C. 2011. Intensidad de uso de hábitat del quirquincho andino (Chaetophractus nationi) en zonas aledañas a asentamientos humanos de la provincia Sur Carangas, Oruro, Bolivia. Edentata 12: 28-35. https://doi. org/10.5537/020.012.0105

Pérez-Zubieta, J. C., A. Selaya, Z. Porcel, L. Torrico \& K. Palenque. 2009. Chaetophractus nationi. Pp. 465-466 in: Libro Rojo de la fauna silvestre de vertebrados de Bolivia (L. F. Aguirre, R. Aguayo, J. A. Balderrama, C. Cortez, T. Tarifa \& O. Rocha O., eds.). Ministerio de Medio Ambiente y Agua, La Paz, Bolivia.

Superina, M., C. Brieva R., R. F. Aguilar \& F. Trujillo. 2014. Manual de mantenimiento y rehabilitación de armadillos. Fundación Omacha, ODL, Cormacarena, Corporinoquia, Corpometa y Bioparque Los Ocarros. Bogotá, Colombia. 96 pp.

Zúñiga, E. \& L. Araya. 2014. Abundancia relativa y patrones de actividad de Chaetophractus nationi (Thomas, 1894), en la Reserva de la Biósfera Lauca. Biodiversidata 2: 35-38.

Recibido: 17 de mayo de 2017; Aceptado: 12 de octubre de 2017 